\title{
Dielectric Properties of Zeolitic Imidazolate Frameworks in the Broadband Infrared Regime
}

\author{
Matthew R. Ryder, ${ }^{1,2,3}$ Zhixin Zeng, ${ }^{1}$ Kirill Titov, ${ }^{1}$ Yueting Sun, ${ }^{1}$ E.M. Mahdi, ${ }^{1}$ Irina Flyagina, ${ }^{1}$ \\ Thomas D. Bennett, ${ }^{4}$ Bartolomeo Civalleri, ${ }^{5}$ Chris S. Kelley, ${ }^{2}$ Mark D. Frogley, ${ }^{2}$ \\ Gianfelice Cinque, ${ }^{2}$ and Jin-Chong Tan ${ }^{1 *}$ \\ ${ }^{1}$ Multifunctional Materials \& Composites (MMC) Laboratory, Department of Engineering Science, University of Oxford, \\ Parks Road, Oxford OX1 3PJ, United Kingdom \\ ${ }^{2}$ Diamond Light Source, Harwell Campus, Chilton, Oxford OX11 0DE, United Kingdom \\ ${ }^{3}$ ISIS Facility, Rutherford Appleton Laboratory, Chilton, Didcot OX11 0QX, United Kingdom \\ ${ }^{4}$ Department of Materials Science and Metallurgy, University of Cambridge, Cambridge CB3 0FS, United Kingdom \\ ${ }^{5}$ Department of Chemistry, NIS and INSTM Reference Centre, University of Turin, via Pietro Giuria 7, 10125 Torino, Italy \\ *Email address: jin-chong.tan@eng.ox.ac.uk
}

\begin{abstract}
:
The field of metal-organic framework (MOF) materials is rapidly advancing towards practical applications, consequently it is urgent to achieve a better understanding and precise control of their physical properties. Yet research on the dielectric properties of MOFs is at its infancy, where studies are confined to the static dielectric behavior or lower frequency response $(\mathrm{kHz}-\mathrm{MHz})$ only. Herein we present the pioneering use of synchrotron-based infrared reflectivity experiments, combined with density functional theory (DFT) calculations to accurately determine the dynamic dielectric properties of zeolitic imidazolate frameworks (ZIFs: a topical family of MOFs). We show, for the first time, the frequency-dependent dielectric response of representative ZIF compounds, bridging the near-, mid-, and far-infrared (terahertz THz) broadband frequencies. We establish the structure-property relations as a function of framework porosity and structural change. Our comprehensive results will be paving the way for novel ZIF-based terahertz applications, such as infrared optical sensors and high-speed wireless communications.
\end{abstract}


TOC graphic $(5 \mathrm{~cm} \times 5 \mathrm{~cm})$

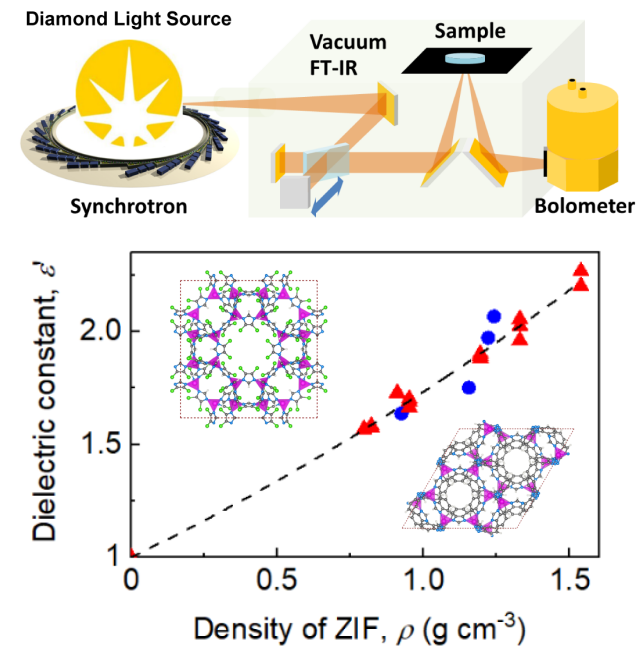


Metal-organic framework (MOF) materials have long been considered as promising candidates for industrial gas storage and gas separations due to their selectivity and significantly large surface areas, in the range of 1,000 to $10,000 \mathrm{~m}^{2} \mathrm{~g}^{-1} .{ }^{1}$ However, large quantities of the materials are required for such applications, and this, along with other restrictions such as poor processing scalability, has resulted in the recent shift in scientific focus to investigate the potential of these hybrid materials to afford smaller value-added applications, such as photonics-based sensing, ${ }^{2,3}$ smart switches, ${ }^{4,5}$ energy conversion devices ${ }^{6}$ and tunable optoelectronics. $^{7,8}$

Materials, such as MOFs, featuring "ultralow- $k$ " dielectric constants $(k \sim 1.1$ to 1.5$)$ are promising candidate materials for the future microelectronics industry. This is due to the dependence of low- $k$ dielectric response upon porosity ${ }^{9}$ and the underlying framework structure, ${ }^{10}$ both of which are tunable in MOF materials. Of specific interest are devices that can lead to integrated circuits, in which the MOF may act as an interlayer dielectric insulating material. ${ }^{7,11}$ There are, however, a number of challenges for the engineering of functional devices integrating new low- $k$ materials to accomplish real-world applications. ${ }^{7,12}$ These include thermal and chemical stability, predictable mechanical behavior, long-term resilience, and excellent adhesion to adjacent device interlayers.

The latest literature highlights the promising future of MOFs as low- $k$ dielectric materials, ${ }^{13}$ though little work has been done focusing on the terahertz (THz) and infrared (IR) frequency domains. ${ }^{10}$ Theoretical work in the field exists but has been mainly limited to the semi-empirical Clausium-Mossitti model, and only applied to the static dielectric constant of cubic Zn-based MOFs. ${ }^{9}$ There have more recently been some interesting $a b$ initio studies on hypothetical frameworks, where qualitative dynamic (frequency-dependent) dielectric constants in the near-ultraviolet (UV) region have been elucidated. ${ }^{14}$ An example of experimental study exists employing spectroscopic ellipsometry to determine the frequency-dependent dielectric and optical properties of HKUST-1 thin films. The results were in reasonable agreement with theory, but again the work reported was restricted to the near-UV region. ${ }^{15}$ Thin films of ZIF- 8 have also been studied in the lower $\mathrm{kHz}$ to $\mathrm{MHz}$ frequency regions employing a capacitor arrangement, highlighting zeolitic imidazolate frameworks (ZIFs) as promising candidates as low- $k$ dielectrics. ${ }^{16}$ Other recent studies within the $\mathrm{kHz}-\mathrm{MHz}$ frequencies have investigated the dielectric properties of another $\mathrm{Zn}$-based MOF in the presence of solvent molecules, illustrating the opportunity to tune host-guest interactions ${ }^{17}$ and an additional $\mathrm{Zn}$-based MOF with high thermal stability. ${ }^{18}$

One major limitation delaying these promising materials for engineering dielectric applications is that experimental methods to characterize these properties across the broadband spectral frequency range are insufficient. Measuring the dielectric constants of polycrystalline pellets is challenging, particularly by employing the conventional capacitor impedance method in the $\mathrm{MHz}$ frequency range, ${ }^{13,16}$ where intercrystal porosity may affect the determined values due to air gaps entrapped in the pellet. Dielectric measurement technique encompassing the $\mathrm{THz}$ spectral region is also less developed, but important for emergent high-speed communication technologies ${ }^{19}$ and optical remote sensing applications. ${ }^{20}$ In addition, theoretical methods are 
only starting to provide an improved procedure to allow for a quantitative determination of the dynamic dielectric behavior of complex framework materials such as MOFs. ${ }^{10}$

Fourier transform infrared (FTIR) spectroscopy has been very informative in probing the structural dynamics of MOFs. We recently demonstrated this using synchrotron-based far-IR spectroscopy and inelastic neutron scattering (INS), in conjunction with ab initio density functional theory (DFT) to explain the framework-specific collective vibrational motions of a variety of MOF structures at the THz frequencies. ${ }^{21,22}$ Intriguingly, these $\mathrm{THz}$ vibrational motions are linked to various physical phenomena, including gate-opening and breathing mechanisms in ZIF-8 and ZIF-7, ${ }^{23,24}$ and shear-driven destabilization motions of ZIF structures. ${ }^{25}$

Synchrotron IR spectroscopy can also be utilized to achieve high-quality reflectance spectra across the broad IR spectral range using a single setup, thereby avoiding systematic errors. By adopting the KramersKronig Transformation (KKT) theory, ${ }^{26}$ the real and imaginary parts of the complex refractive index as a function of frequency, $\tilde{n}(\omega)$, can be determined as:

$$
\tilde{n}(\omega)=n(\omega)+i \kappa(\omega)
$$

While the dynamic dielectric constant, or more precisely the real and imaginary parts of the frequencydependent complex dielectric function, $\tilde{\varepsilon}(\omega)$, can be determined as:

$$
\tilde{\varepsilon}(\omega)=\varepsilon^{\prime}(\omega)+i \varepsilon^{\prime \prime}(\omega)
$$

It is noted that $\tilde{n}(\omega)$ and $\tilde{\varepsilon}(\omega)$ are dependent on each other as a direct result of the causality principle. The relationship between these two complex physical quantities is:

$$
\tilde{n}^{2}=\tilde{\varepsilon}
$$

where the real and imaginary parts of the dielectric constant as a function of frequency $\omega$, are given by:

$$
\begin{gathered}
\varepsilon^{\prime}(\omega)=n^{2}-\kappa^{2} \\
\varepsilon^{\prime \prime}(\omega)=2 n \kappa
\end{gathered}
$$

The dynamic dielectric constants presented in this study have been calculated using the KKT routine as implemented in the Bruker OPUS code. ${ }^{27}$

In this study, for the first time, we report the broadband frequency-dependent dielectric and optical properties for a topical family of ZIF materials (see Figure 1). We demonstrate the efficacy of the synchrotronbased reflectance spectroscopy technique (Figure 2) to accomplish accurate dynamic dielectric measurements, bridging the near-, mid-, and far-infrared broadband frequencies (Figure 3), hitherto unexplored in the MOF literature today. Subsequently, we established the structure-property relationships underpinning the ZIF family of materials, elucidating the fundamental roles played by porosity and structural variation on the tunability of dielectric properties. The new methodologies we proposed have addressed outstanding challenges in the field, specifically by opening the door to the quantitative characterization of numerous MOF structures also transferable to novel functional compounds, the majority of which are only obtainable in polycrystalline fine powder forms reminiscent of the samples employed in this work. 


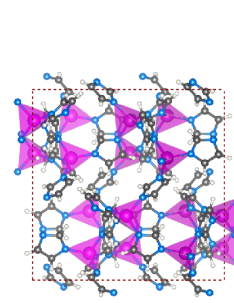

(a) ZIF-4

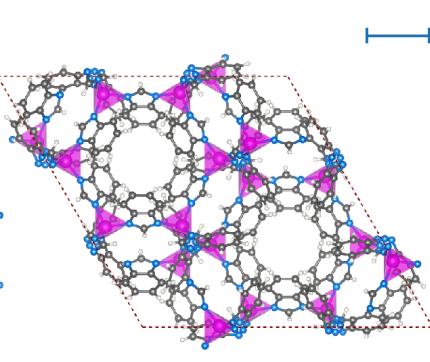

(b) ZIF-7

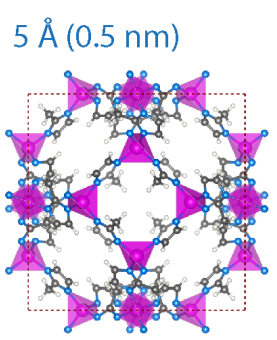

(c) ZIF-8

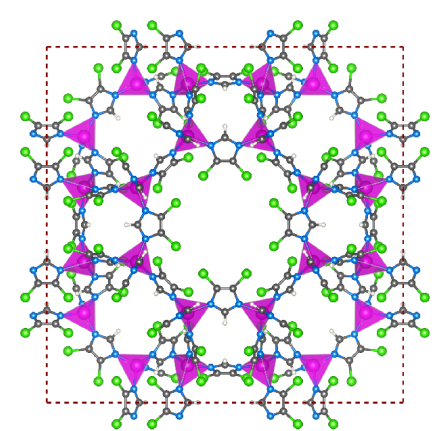

(d) ZIF-71

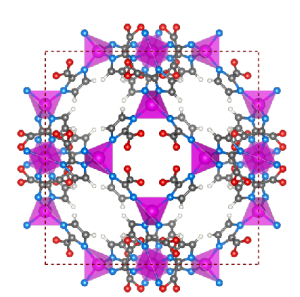

(e) ZIF-90

Figure 1. Porous framework structures of (a) ZIF-4, (b) ZIF-7, (c) ZIF-8, (d) ZIF-71 and (e) ZIF-90, viewed along the crystallographic $c$-axis. The ZIF structures named above are constructed from the same inorganic building unit: $\mathrm{ZnN}_{4}$ tetrahedra (highlighted in purple) bridged by a series of different imidazolate-derived organic linkers: imidazolate, 2-benzimidazolate, 2-methylimidazolate, 4,5-dichloroimidazolate, and 2-carboxyaldehyde imidazolate (note that the $\mathrm{O}$ atoms are disordered in the ZIF-90 linkers), respectively. Dashed lines represent a one unit cell. Color scheme: $\mathrm{Zn}$ pink; $\mathrm{C}$ gray; $\mathrm{N}$ blue; $\mathrm{O}$ red; $\mathrm{Cl}$ green; $\mathrm{H}$ white. Sample preparation details are given in the Supporting Information (SI section 1).

The experimental spectra were measured using a customized setup at the Diamond Light Source (Oxfordshire, UK) utilizing a Bruker Vertex 80V FTIR interferometer, to provide in situ IR data using a synchrotron radiation source. The advantage is the ability to use specular reflectance technique which enables the KKT calculations and the broadband of the synchrotron radiation to cover the whole IR spectral range. A more detailed description of the experimental setup is available in the Supporting Information (see SI section 2). KKT is valid and produces reliable results under specific conditions. If the material is absorbing infrared radiation (as is the case with most materials, including MOFs), then the transformed spectra will be affected by the absorption bands, and it is, therefore, critical to ensure that the selected spectral cut-offs for the KKT analysis do not coincide with any absorption features. This is not a major concern towards the near-IR region, as overtone absorptions are very weak. However, care must be taken when selecting the spectral cut-off in the mid-IR and far-IR regions, where there are many distinctive absorption features present in MOFs. ${ }^{25,28}$

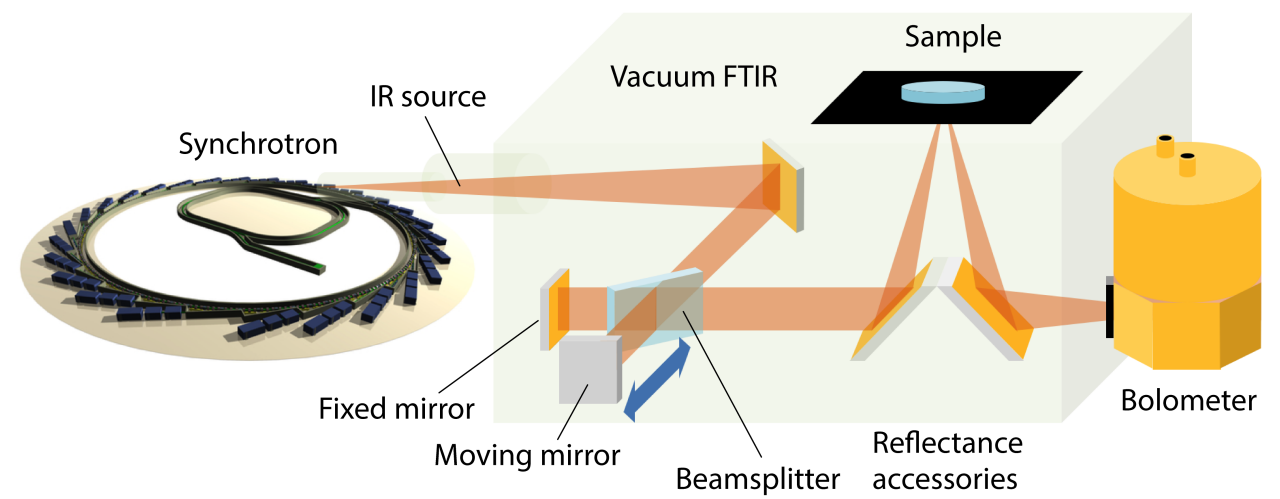

Figure 2. Schematic of the experimental setup employed in the synchrotron-based reflectivity measurements conducted at the B22 MIRIAM Beamline in the Diamond Light Source (Oxfordshire, UK). 
Specular reflection occurs at the sample surface, thereby requiring the samples to exhibit a low roughness value $(<100 \mathrm{~nm})$ compared to the incident wavelength. The reflectance spectra in this study were collected using pressed pellets of ZIF powder materials ( $\sim 1 \mathrm{~mm}$ thick with $13 \mathrm{~mm}$ diameter), where we have ensured that the prepared pellets were as smooth as was practically possible. The surface quality was characterized using the Alicona InfiniteFocus 3D profilometer (non-contact mode), which confirmed that the root-meansquare (RMS) roughness of the pellets was below $50 \mathrm{~nm}$. Additionally, we found that surface roughness was not strongly affected by the magnitude of pressure used to press the specific pellet (under 1-10 tonnes, see SI section 3).

Figure 3 shows the experimental frequency-dependent dielectric properties of ZIF materials in the extended infrared (IR) spectral region encompassing $65-9000 \mathrm{~cm}^{-1}(\sim 2-270 \mathrm{THz})$, determined from the measured reflectivity spectra (see SI section 4). The dynamic dielectric data were obtained for the five ZIF structures (Figure 1), namely: ZIF-4, ZIF-7, ZIF-8, ZIF-71 and ZIF-90, which are all Zn-based materials with different imidazolate derived linkers. We found that all dielectric constants, $\varepsilon^{\prime}(\omega)$, are lying within the range of 1.22.5. Of particular interest are the relatively low values witnessed closer to the near-IR spectral region, i.e. beyond $4000 \mathrm{~cm}^{-1}\left(>120 \mathrm{THz}\right.$ ). The values of $\varepsilon^{\prime}(\omega)$ are $\sim 1.2$ for all the structures, excluding ZIF-90 (likely due to the microstructural defects in ZIF-90). ${ }^{29}$ Moreover, we observed that the near-IR spectral region ( $>4000$ $\mathrm{cm}^{-1}$ ) appears to be less sensitive to the framework structure, exhibiting an overall common asymptotic behavior. The corresponding values of the complex refractive index of the five ZIF structures, i.e. $n(\omega)$ and $\kappa(\omega)$ as a function of frequency, are summarized in the Supporting Information (SI section 5). In fact, the dependence on the structure is still relevant even in the higher frequency regions of $270 \mathrm{THz}\left(9000 \mathrm{~cm}^{-1}\right)$. However, in this study we found that for ZIFs it is significantly less structure-dependent in the near-infrared frequencies $\left(>4000 \mathrm{~cm}^{-1}\right)$ compared to the lower THz frquencies $\left(<600 \mathrm{~cm}^{-1}\right)$ or static dielectric values. 
$\mathrm{THz}$
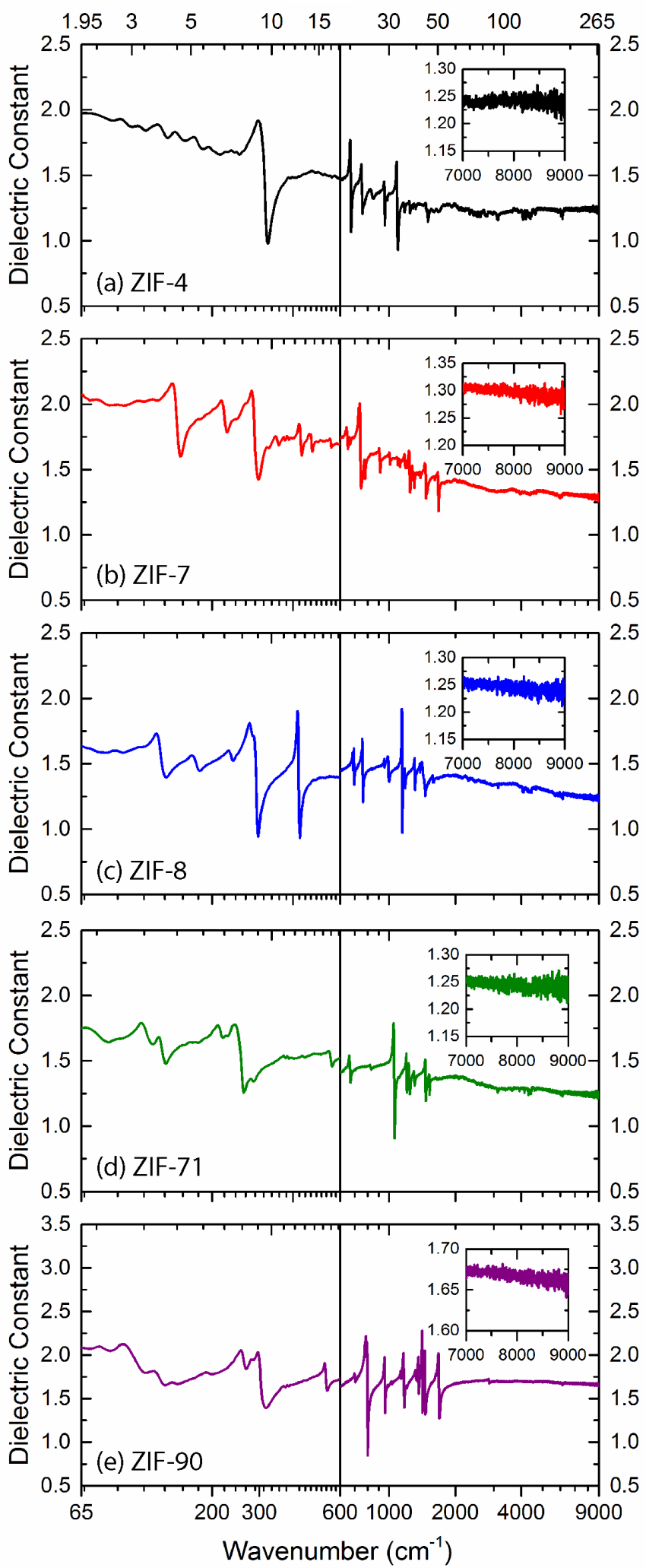

Figure 3. Frequency-dependent dielectric functions of (a) ZIF-4, (b) ZIF-7, (c) ZIF-8, (d) ZIF-71 and (e) ZIF-90, measured across the far-IR and mid-IR spectral regions. The real part of the dielectric constants plotted here corresponds to the magnitude of $\varepsilon^{\prime}(\omega)$, obtained from the Kramers-Kronig Transformation (KKT). Given the broadband data, the farIR and mid-IR regions are both plotted on separate logarithmic axes for clarity. The spectral region at the boundary between the mid-IR and near-IR $\left(7000-9000 \mathrm{~cm}^{-1}\right)$ is shown as an inset to show the dielectric values more clearly. 
The spectral data in Figure 3 reveal that the dielectric values decline with increasing frequency, suggesting that the static dielectric constant, $\varepsilon^{\prime}(\omega=0)$, represents the maximum value for each of the ZIF materials. Also, the gradient in the decrease of the dielectric constant with respect to wavenumber is higher in the low frequency far-IR region, compared with the mid- and near-IR (Figure 4). The data presented in Figures 3 and 5 demonstrate that the far-IR dynamic dielectric constants are significantly structure dependent and show a direct link to the porosity and the density of the framework. The direct link to the level of porosity is to be expected from a fundamental point-of-view, as the dielectric constant is a measure of the ability of a substance to store electrical energy in an electric field, such that when the porosity within the framework increases the dielectric constant $\varepsilon^{\prime}$ will tend towards the value of a vacuum $\left(\varepsilon^{\prime}=1\right)$ or air $\left(\varepsilon^{\prime} \sim 1\right)$.

Because the specular reflectance experiments were conducted on pelletized samples, the effects of pelletization pressure were investigated to ensure that accurate dielectric constant values were being reported and to ensure that no pressure-induced amorphisation or phase transitions, as often reported for the ZIF family, had occurred. ${ }^{30}$ We performed an extensive investigation into the effects of pressure on the ZIF- 8 material. Figure 4(a) shows the frequency-dependent dielectric function of ZIF-8 predicted by ab initio density functional theory (DFT) using the CRYSTAL14 $\operatorname{code}^{31}$ (see computational details in SI section 6). Notably all major dielectric transitions have been correctly identified, although the magnitudes of the experimental dielectric constant (Figure 4(b)) are relatively lower than the DFT theoretical values predicted for an idealized single crystal. This discrepancy may be linked to the polycrystalline nature of the pellets ${ }^{10}$ used in the experiments, where intercrystal porosity may affect the determined values due to air gaps entrapped in the pelletized sample.

As can be seen from Figure 4(c-f), our data suggest that there is a linear trend in the increase of the dielectric constant at each spectral region with increased pelletization pressure. Noteworthy, the pelletization pressure had a greater effect on the far-IR spectral region $(\mathrm{THz})$ with an increased gradient of $0.71 \mathrm{GPa}^{-1}$, for example this is more than doubled that of the near-IR region (gradient $=0.35 \mathrm{GPa}^{-1}$ ). The increase in dielectric constant was confirmed to be primarily an effect of the porous structure mechanically straining under the pelletization pressure $^{32}$ and an additional contribution resulting from the higher densification of the pellet. This was confirmed via X-ray diffraction (XRD), linking the increase in dielectric constants to the decrease in the Bragg peak intensities of ZIF-8 (see XRD data in SI Figure S8). We observed the onset of the pressure-induced phase transition of ZIF-8 at pressures above $0.3 \mathrm{GPa}$, indicated by the evolution of a new spectral feature at $\sim 385$ $\mathrm{cm}^{-1}$ (also SI Figure S21 inset). However, the spectral feature observed around $420-450 \mathrm{~cm}^{-1}$ is a numerical artefact resulting from the spectral range used for the KKT; the artefact can be eliminated as discussed in the SI section 7. 

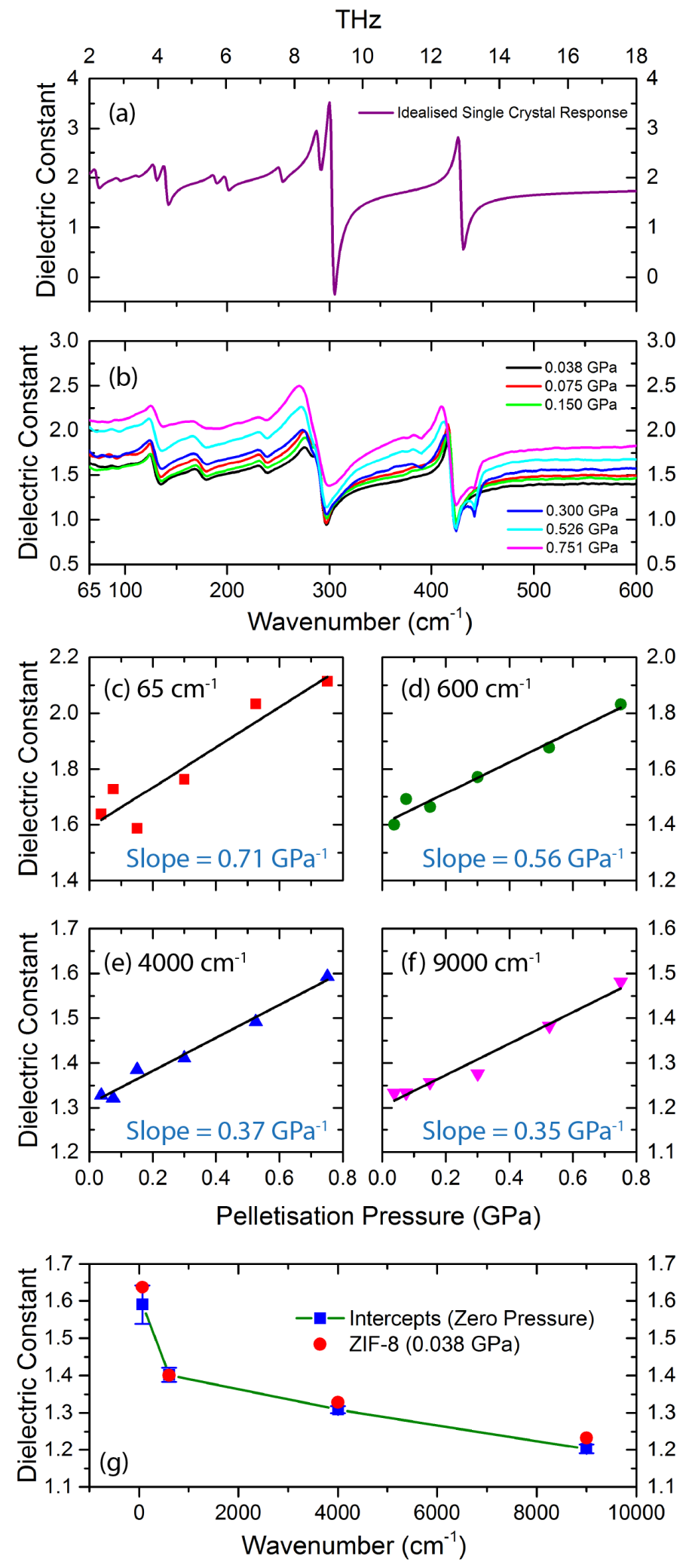

Figure 4. Spectra of the (a) theoretical DFT and (b) experimental far-IR dielectric data of ZIF-8 showing the stepwise effect of pelletization pressure on the real part of the dielectric constant, i.e. $\varepsilon^{\prime}(\omega)$. Plots showing the upward trend in the dielectric constant of ZIF-8, upon increased pelletization pressure, at a range of specific spectral points: (c) $65 \mathrm{~cm}^{-1}$; (d) $600 \mathrm{~cm}^{-1}$; (e) $4000 \mathrm{~cm}^{-1}$; (f) $9000 \mathrm{~cm}^{-1}$. The gradient of the fitted trend line highlights that the dielectric constant is more sensitive to increased palletization pressure in the THz-frequency region $\left(1.95 \mathrm{THz} \approx 65 \mathrm{~cm}^{-1}\right)$. (g) The dielectric constant values for ZIF-8 at $65,600,4000$ and $9000 \mathrm{~cm}^{-1}$, extrapolated to assume zero pressure effects. 

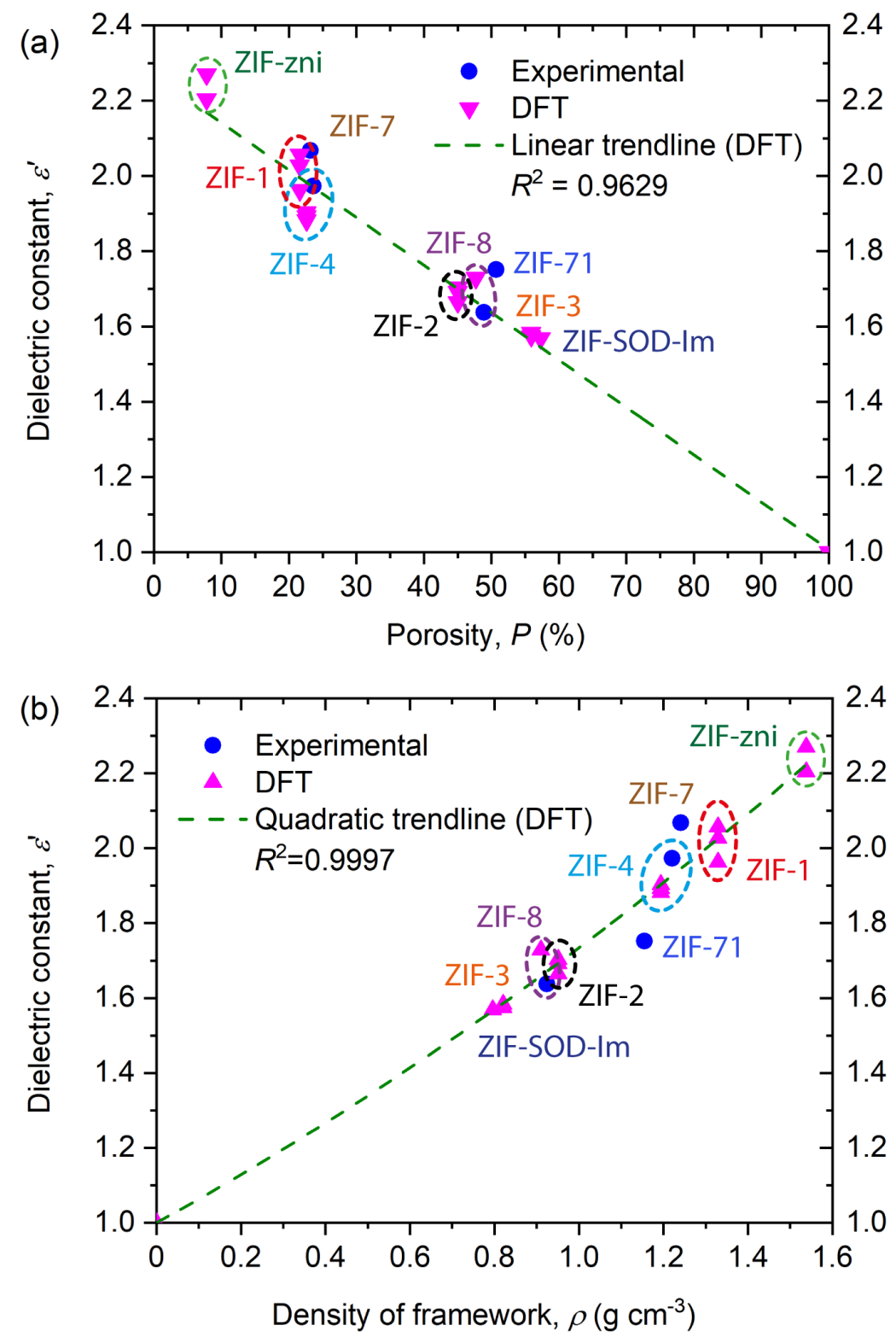

Figure 5. Trends observed in the THz-frequency dielectric constant $\mathcal{E}^{\prime}$ (at $1.95 \mathrm{THz} \approx 65 \mathrm{~cm}^{-1}$ ) presented as a function of porosity $(P)$ and framework density $(\rho)$. (a) Linear trend $\varepsilon^{\prime}=\mathrm{A} P+\mathrm{B}$, where $\mathrm{A}=-0.01261$ and $\mathrm{B}=2.26726$. (b) Quadratic trend $\varepsilon^{\prime}=\mathrm{C} \rho^{2}+\mathrm{D} \rho+\mathrm{E}$, where $\mathrm{C}=0.11276, \mathrm{D}=0.62165, \mathrm{E}=1$. The theoretical DFT values were obtained from optimized ZIF structures at the B3LYP-D ${ }^{*}$ level of theory (computational details in SI section 6, and in accordance with Refs. ${ }^{10,33}$ ), corresponding to the values of dielectric constants along the three orthonormal axes $(x, y, z)$. The multiple values represent the anisotropic single-crystal values for all the non-cubic unit cell structures. For example, both ZIF-8 and ZIF-SOD-Im each has one unique dielectric constant value only because of cubic symmetry (where $a=b=c$ ) thus the dielectric constant along $x=y=z$. In contrast, ZIF-zni has two unique values because it has a tetragonal unit cell, therefore its dielectric constant value along $x=y \neq z$. The other structures such as ZIF-1 (monoclinic), ZIF-2 and ZIF-4 (orthorhombic), ZIF-3 (tetragonal), each has three unique values since the dielectric constant in $x \neq y \neq z$. The theoretical dielectric constants are static values, i.e. $\omega=0$, thus showing some discrepancies to the experimental values measured at $65 \mathrm{~cm}^{-1}$. Note: The porosity and density values were determined from the Crystallographic Information Files (CIFs) using the Mercury CSD program. ${ }^{34}$ ZIFs-1 to -4 and ZIF-zni have the same chemical composition Zn(imidazolate) $)_{2}$ but exhibit different topology and porosity, details of the structures can be found in Refs. ${ }^{35,36}$. The ZIF-SOD-Im structure is a hypothetical sodalite (SOD) framework akin to ZIF-8, formulated by substituting the 2-methylimidazolate linkers of ZIF-8 with the (less bulky) unsubstituted imidazolate (Im) linkers to yield a more porous structure with reduced density. 
We found that pellets compressed under 0.038-0.150 GPa of pressure (i.e. 0.5-2.0 ton over a 13-mm diameter pellet) did not affect the resultant dielectric values significantly, as can be seen from Figure 4(g), values obtained using these pellets were therefore used to analyze structural trends over the broader ZIF series. Figure 5 shows the structure-property correlation we established between the $\mathrm{THz}$ region $\left(65 \mathrm{~cm}^{-1}\right)$ dielectric constant with the framework porosity, and with the physical density of the framework. In establishing the trends in Figure 5, we have further considered the DFT theoretical static values (i.e. effectively zero frequency) of additional ZIF structures: ZIF-1, -2, -3, -4 while the upper and lower bounds in the current study are defined by ZIF-zni and the hypothetical ZIF-SOD-Im structure, respectively. It can be seen that the comparison between the DFT values (static) and the experimental values $(\mathrm{THz})$ is reasonably good. This result, therefore, allows us to establish a first estimate of the THz-frequency dielectric constant (likewise, the refractive index) of other ZIF structures by the structure-property trends projected in Figure 5.

While all the dielectric constant values considered in this study are for the Zn-based materials, it can be anticipated that the effect of the specific metal node to be less significant than the role of the bridging organic linkers. ${ }^{14,33,37}$ On this basis, we consider the primary contributing factors to the dielectric constant are strongly dependent upon the levels of porosity scaling with the framework density. If we extrapolate the trend to a porosity value of $100 \%$, it will result in a dielectric constant of $\sim 1$ (this value is to be expected as the porosity of $100 \%$ is essentially air). Therefore, if we consider some of the most porous MOFs, when completely activated, then we can predict dielectric constants of approximately the same response to air. For example, we reasoned that the Zn-based MOF with the lowest density obtained from the hypothetical MOF database, ${ }^{38}$ would give a dielectric constant of $\sim 1.1$ (density $=0.118 \mathrm{~g} \mathrm{~cm}^{-3}$ ). Furthermore, if we may assume that the choice of metal or linker does not make a major difference then the recently synthesized and lowest-density MOF to be experimentally proven, uranium-based NU-1301 (density $\left.=0.124 \mathrm{~g} \mathrm{~cm}^{-3}\right)^{39}$ would also have a static dielectric constant of $\sim 1.1$. The exact value, however, will require further experimental validations.

In summary, this study demonstrates the pioneering use of specular reflectance spectroscopic experiments to systematically obtain complex dielectric functions and refractive index of ZIFs in the extended infrared (IR) region (65-9000 $\left.\mathrm{cm}^{-1}\right)$. Of particular novelty are the $\mathrm{THz}$ region dielectric response and the good agreement with theoretical values calculated via DFT. The dynamic dielectric behavior of the ZIF structures was analyzed, and the THz-frequency dielectric constants were found to be structure-dependent and exhibiting a strong correlation with the porosity and the density of the open framework. The structure-property trend derived in this work can be exploited to motivate research concerned with the engineering of tunable MOF dielectrics. It will set the foundation for the promising field of ultralow- $k$ porous materials and subsequent integration into a host of polycrystalline thin-film devices. ${ }^{7,40,41}$ 


\section{Supporting Information (SI) Available:}

Materials synthesis and characterization methods; Experimental setup and analysis of reflectivity data; surface characterization; Reflectivity spectra; Real and imaginary parts of the complex dielectric constant and complex refractive index; Ab initio density functional theory calculations; ZIF-8 spectral features and corrections.

\section{Acknowledgements}

We acknowledge the Diamond Light Source for the provision of beamtime no. SM10215 and SM14902 at B22 MIRIAM. M.R.R. would like to thank the UK Engineering and Physical Sciences Research Council (EPSRC) for a DTA postgraduate scholarship and the Science and Technology Facilities Council (STFC) CMSD Award 13-05. M.R.R. would also like to thank the EPSRC for a Doctoral Prize Fellowship and the Rutherford Appleton Laboratory (RAL) for access to the SCARF cluster and additional computing resources. T.D.B. would like to thank the Royal Society for a University Research Fellowship. J.C.T. acknowledged the EPSRC for research funding (EP/N014960/1). We are grateful to the ISIS Support Laboratories, especially Dr. Marek Jura and Dr. Gavin Stenning at the Materials Characterisation Laboratory (R53) for access to the X-ray diffraction equipment. We thank the Research Complex at Harwell (RCaH) for the provision of advanced materials characterization suite. 


\section{References}

(1) Furukawa, H.; Cordova, K. E.; O'Keeffe, M.; Yaghi, O. M. The chemistry and applications of metalorganic frameworks. Science 2013, 341, 1230444.

(2) Lustig, W. P.; Mukherjee, S.; Rudd, N. D.; Desai, A. V.; Li, J.; Ghosh, S. K. Metal-organic frameworks: Functional luminescent and photonic materials for sensing applications. Chem. Soc. Rev. 2017, 46, 3242-3285.

(3) Chaudhari, A. K.; Kim, H. J.; Han, I.; Tan, J. C. Optochemically responsive 2D nanosheets of a 3D metalorganic framework material. Adv. Mater. 2017, 29, 1701463.

(4) Dolgopolova, E. A.; Shustova, N. B. Metal-organic framework photophysics: Optoelectronic devices, photoswitches, sensors, and photocatalysts. MRS Bull. 2016, 41, 890-896.

(5) Ryder, M. R.; Tan, J. C. Nanoporous metal-organic framework materials for smart applications. Mater. Sci. Tech. 2014, 30, 1598-1612.

(6) Zhang, H.; Nai, J.; Yu, L.; Lou, X. W. Metal-organic-framework-based materials as platforms for renewable energy and environmental applications. Joule 2017, 1, 77-107.

(7) Stassen, I.; Burtch, N.; Talin, A.; Falcaro, P.; Allendorf, M.; Ameloot, R. An updated roadmap for the integration of metal-organic frameworks with electronic devices and chemical sensors. Chem. Soc. Rev. 2017, $46,3185-3241$.

(8) Stavila, V.; Talin, A. A.; Allendorf, M. D. MOF-based electronic and opto-electronic devices. Chem. Soc. Rev. 2014, 43, 5994-6010.

(9) Zagorodniy, K.; Seifert, G.; Hermann, H. Metal-organic frameworks as promising candidates for future ultralow-k dielectrics. Appl. Phys. Lett. 2010, 97, 251905.

(10) Titov, K.; Zeng, Z.; Ryder, M. R.; Chaudhari, A. K.; Civalleri, B.; Kelley, C. S.; Frogley, M. D.; Cinque, G.; Tan, J. C. Probing dielectric properties of metal-organic frameworks: MIL-53(Al) as a model system for theoretical predictions and experimental measurements via synchrotron far- and mid-infrared spectroscopy. $J$. Phys. Chem. Lett. 2017, 8, 5035-5040.

(11) Usman, M.; Mendiratta, S.; Lu, K. L. Metal-organic frameworks: New interlayer dielectric materials. ChemElectroChem 2015, 2, 786-788.

(12) Volksen, W.; Miller, R. D.; Dubois, G. Low dielectric constant materials. Chem. Rev. 2010, 110, 56-110.

(13) Mendiratta, S.; Usman, M.; Lu, K.-L. Expanding the dimensions of metal-organic framework research towards dielectrics. Coord. Chem. Rev. 2018, 360, 77-91.

(14) Warmbier, R.; Quandt, A.; Seifert, G. Dielectric properties of selected metal-organic frameworks. $J$. Phys. Chem. C 2014, 118, 11799-11805.

(15) Redel, E.; Wang, Z.; Walheim, S.; Liu, J.; Gliemann, H.; Woell, C. On the dielectric and optical properties of surface-anchored metal-organic frameworks: A study on epitaxially grown thin films. Appl. Phys. Lett. 2013, 103, 091903.

(16) Eslava, S.; Zhang, L.; Esconjauregui, S.; Yang, J.; Vanstreels, K.; Baklanov, M. R.; Saiz, E. Metalorganic framework ZIF-8 films as low- $\kappa$ dielectrics in microelectronics. Chem. Mater. 2013, 25, 27-33.

(17) Yu, S. S.; Yuan, G. J.; Duan, H. B. The low dielectric constant and relaxation dielectric behavior in hydrogen-bonding metal-organic frameworks. RSC Adv. 2015, 5, 45213-45216. 
(18) Mendiratta, S.; Usman, M.; Chang, C.-C.; Lee, Y.-C.; Chen, J.-W.; Wu, M.-K.; Lin, Y.-C.; Hsu, C.-P.; Lu, K.-L. Zn(II)-based metal-organic framework: An exceptionally thermally stable, guest-free low dielectric material. J. Mater. Chem. C 2017, 5, 1508-1513.

(19) Nagatsuma, T.; Ducournau, G.; Renaud, C. C. Advances in terahertz communications accelerated by photonics. Nat. Photonics 2016, 10, 371-379.

(20) Dhillon, S. S.; Vitiello, M. S.; Linfield, E. H.; Davies, A. G.; Hoffmann, M. C.; Booske, J.; Paoloni, C.; Gensch, M.; Weightman, P.; Williams, G. P., et al. The 2017 terahertz science and technology roadmap. $J$. Phys. D-Appl. Phys. 2017, 50, 043001.

(21) Ryder, M. R.; Van de Voorde, B.; Civalleri, B.; Bennett, T. D.; Mukhopadhyay, S.; Cinque, G.; Fernandez-Alonso, F.; De Vos, D.; Rudić, S.; Tan, J.-C. Detecting molecular rotational dynamics complementing the low-frequency terahertz vibrations in a zirconium-based metal-organic framework. Phys. Rev. Lett. 2017, 118, 255502.

(22) Ryder, M. R.; Civalleri, B.; Cinque, G.; Tan, J. C. Discovering connections between terahertz vibrations and elasticity underpinning the collective dynamics of the HKUST-1 metal-organic framework. CrystEngComm 2016, 18, 4303-4312.

(23) Moggach, S. A.; Bennett, T. D.; Cheetham, A. K. The effect of pressure on ZIF-8: Increasing pore size with pressure and the formation of a high-pressure phase at $1.47 \mathrm{GPa}$. Angew. Chem. Int. Ed. 2009, 48, 70877089.

(24) Aguado, S.; Bergeret, G.; Titus, M. P.; Moizan, V.; Nieto-Draghi, C.; Bats, N.; Farrusseng, D. Guestinduced gate-opening of a zeolite imidazolate framework. New J. Chem. 2011, 35, 546-550.

(25) Ryder, M. R.; Civalleri, B.; Bennett, T. D.; Henke, S.; Rudić, S.; Cinque, G.; Fernandez-Alonso, F.; Tan, J. C. Identifying the role of terahertz vibrations in metal-organic frameworks: From gate-opening phenomenon to shear-driven structural destabilization. Phys. Rev. Lett. 2014, 113, 215502.

(26) Lucarini, V.; Saarinen, J. J.; Peiponen, K. E.; Vartiainen, E. M. Kramers-Kronig relations in optical materials research. Springer-Verlag: Berlin Heidelberg; 2005.

(27) Bruker. OPUS spectroscopy software (version 7), 2017, https://www.bruker.com/products/infrared-nearinfrared-and-raman-spectroscopy/opus-spectroscopy-software.html.

(28) Ryder, M. R.; Bennett, T. D.; Kelley, C. S.; Frogley, M. D.; Cinque, G.; Tan, J. C. Tracking thermalinduced amorphization of a zeolitic imidazolate framework via synchrotron in situ far-infrared spectroscopy. Chem. Commun. 2017, 53, 7041-7044.

(29) Flyagina, I. S.; Mahdi, E. M.; Titov, K.; Tan, J. C. Thermo-mechanical properties of mixed-matrix membranes encompassing zeolitic imidazolate framework-90 and polyvinylidine difluoride: ZIF-90/PVDF nanocomposites. APL Mater. 2017, 5, 086104.

(30) Chapman, K. W.; Halder, G. J.; Chupas, P. J. Pressure-induced amorphization and porosity modification in a metal-organic framework. J. Am. Chem. Soc. 2009, 131, 17546-17547.

(31) Dovesi, R.; Orlando, R.; Erba, A.; Zicovich-Wilson, C. M.; Civalleri, B.; Casassa, S.; Maschio, L.; Ferrabone, M.; De La Pierre, M.; D'Arco, P., et al. CRYSTAL14: A program for the ab initio investigation of crystalline solids. Int. J. Quantum Chem. 2014, 114, 1287-1317.

(32) Zeng, Z.; Tan, J. C. AFM nanoindentation to quantify mechanical properties of nano- and micron-sized crystals of a metal-organic framework material. ACS Appl. Mater. Interfaces 2017, 9, 39839-39854.

(33) Ryder, M. R.; Donà, L.; Vitillo, J. G.; Civalleri, B. Understanding and controlling the dielectric response of metal-organic frameworks. ChemPlusChem 2018, (In Press, DOI: 10.1002/cplu.201700558). 
(34) Macrae, C. F.; Bruno, I. J.; Chisholm, J. A.; Edgington, P. R.; McCabe, P.; Pidcock, E.; RodriguezMonge, L.; Taylor, R.; van de Streek, J.; Wood, P. A. Mercury CSD 2.0 - new features for the visualization and investigation of crystal structures. J. Appl. Crystallogr. 2008, 41, 466-470.

(35) Ryder, M. R.; Tan, J. C. Explaining the mechanical mechanisms of zeolitic metal-organic frameworks: Revealing auxeticity and anomalous elasticity. Dalton Trans. 2016, 45, 4154-4161.

(36) Tan, J. C.; Civalleri, B.; Erba, A.; Albanese, E. Quantum mechanical predictions to elucidate the anisotropic elastic properties of zeolitic imidazolate frameworks: ZIF-4 vs. ZIF-zni. CrystEngComm 2015, 17, 375-382.

(37) Tan, J. C.; Bennett, T. D.; Cheetham, A. K. Chemical structure, network topology, and porosity effects on the mechanical properties of zeolitic imidazolate frameworks. Proc. Natl. Acad. Sci. USA 2010, 107, 99389943.

(38) Wilmer, C. E.; Leaf, M.; Lee, C. Y.; Farha, O. K.; Hauser, B. G.; Hupp, J. T.; Snurr, R. Q. Large-scale screening of hypothetical metal-organic frameworks. Nat. Chem. 2012, 4, 83-89.

(39) Li, P.; Vermeulen, N. A.; Malliakas, C. D.; Gomez-Gualdron, D. A.; Howarth, A. J.; Mehdi, B. L.; Dohnalkova, A.; Browning, N. D.; O'Keeffe, M.; Farha, O. K. Bottom-up construction of a superstructure in a porous uranium-organic crystal. Science 2017, 356, 624-627.

(40) Buchan, I.; Ryder, M. R.; Tan, J. C. Micromechanical behavior of polycrystalline metal-organic framework thin films synthesized by electrochemical reaction. Cryst. Growth Des. 2015, 15, 1991-1999.

(41) Li, W. J.; Liu, J.; Sun, Z. H.; Liu, T. F.; Lu, J.; Gao, S. Y.; He, C.; Cao, R.; Luo, J. H. Integration of metal-organic frameworks into an electrochemical dielectric thin film for electronic applications. Nat. Commun. 2016, 7, 11830. 